\title{
Complete Gradient-LC-ESI System on a ChIP for Protein ANalysis
}

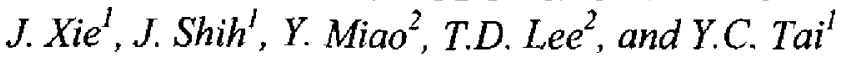 \\ ${ }^{1}$ California Institute of Technology, Pasadena, California, USA \\ ${ }^{2}$ Beckman Research Institute, Duarte, California, USA
}

\begin{abstract}
This paper presents the first fully integrated gradientelution liquid chromatography-electrospray ionization (LC-ESI) system on a chip. This chip integrates a pair of high-pressure gradient pumps, a sample injection pump, a passive mixer, a packed separation column, and an ESI nozzle. We also present the successful on-chip separation of protein digests by reverse phase (RP)-LC coupled with on-line mass spectrometer (MS) analysis.
\end{abstract}

\section{INTRODUCTION}

LC has remained the workhorse of proteomics and miniaturization of LC is essential to meet the demands of fast growing proteomic research and development $[1,2]$. This is evidenced by the trends exhibited by the state of the art in LC, which have been towards smaller beads, smaller diameter columns, and correspondingly lower flow rates. Together, these trends have lead to higher resolution, increased sensitivity, and faster separations. MEMS is ideal for realizing this need for miniaturization.

There are also many advantages to integrating the complete system onto a single chip. Perhaps most importantly, dead volume can be virtually eliminated from the LC system. In desktop LC systems, the necessary equipment is large and requires numerous fittings and tubes. The fluidic connections contribute to a sizable dead volume which increases the time necessary for separation and could possibly affect the separation quality as well. With everything optimally integrated on a single chip, delays associated with dead volume could virtually be eliminated, allowing separations to be completed much more quickly. Another advantage is the possibility of integrating on-chip sensors. This includes, but isn't limited to, sensors which measure flow rate and pressure. The ability to integrate sensors seamlessly within an LC system could in theory produce devices which have higher reliability and better control than conventional systems being used today. Miniaturization and integration of the entire system also has the obvious ability to create smaller systems thus making them portable. The decrease in size often leads to a decrease in power consumption as well, further enhancing the possibility of portable systems.

In this study, we present separations utilizing gradient elutions, as opposed to isocratic elutions. Here, elution refers to the mobile phase being pumped through

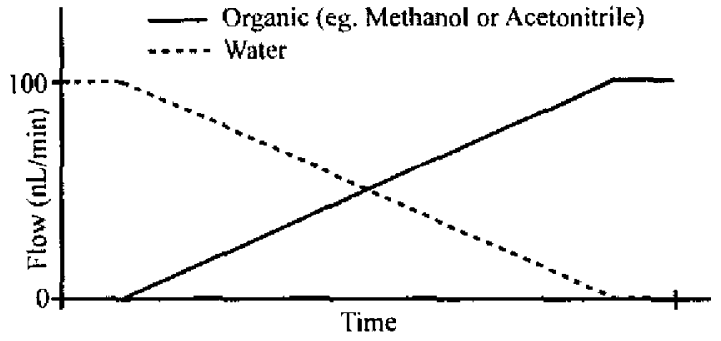

Figure 1: A constant flow rate linear gradient going from $0 \%$ organic to $100 \%$ organic. Typical applications often only require swings from $0 \%$ to $50-70 \%$ depending on the type of sample and organic solvent being used. Optimal flow rates can also vary depending on the column dimensions.

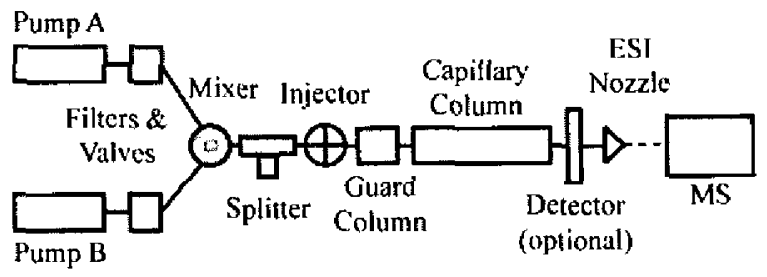

Figure 2: The typical components in a gradient-LC-ESI system. For our particular application we are coupling to a MS using ESI.

the column. In an isocratic elution, the mobile phase composition is fixed, while in a gradient elution, the mobile phase composition is varied during the separation. In the case of RP-LC, where we have a highly hydrophobic stationary phase, we start with a mobile phase of high aqueous concentration and slowly increase the organic concentration as the separation continues. In general, the organic component is a solvent such as acetonitrile or methanol. A typical gradient is shown in Figure 1. In practice, this technique allows the analytes to be separated based on their hydrophobicity. Gradient elutions are particularly useful when the components of a sample exhibit a very large range of hydrophobicity, which is often the case in proteomics. The same sample analyzed using an isocratic elution would result in an extremely long analysis time or incomplete separation.

The components of a typical gradient-LC-ESI system are shown in Figure 2. The basic operation is straightforward. First, sample is injected onto the column. After the sample is injected, a gradient is passed through the column, during which the analytes will elute off the column in order of increasing hydrophobicity. The 
diagrammed system couples to a MS for detection by means of an ESI nozzle. Several other detection methods are also available, such as UV or electrochemical detection, but these methods have the disadvantage of not providing any information about the mass.

The majority of work regarding on-chip separations has been electrophoresis-based. Agilent Technologies has demonstrated a microfabricated column/ESI nozzle using laser ablation of Kapton [3]. But this work lacks on-chip pumping. We have previously demonstrated an ion-exchange LC-on-a-chip with an integrated column and conductivity detection [4], which also lacked integrated pumps, mixers, and the ability to couple to a MS. Perhaps the greatest barrier preventing the total integration of chip-based LC systems is the difficulty in integrating micro-scale high-pressure pumping systems. We recently demonstrated a highpressure pump based on electrolysis [5], which we utilize here.

\section{Design}

The design of our chip mirrors the typical LC-ESI system shown in Figure 2. We incorporate all the essential features onto a $1 \times 2 \mathrm{~cm}$ chip. A picture of our fabricated chip is shown in Figure 3. We incorporate three pumps/reservoirs on our chip, one for a highly aqueous solution, another for a high organic solution, and finally one for our sample. The driving force for these pumps is the pneumatic pressure generated by electrolysis of the aqueous solutions. The bulk of the volume of our reservoirs is attained with the chip packaging which will be described in detail in a later section. In all, each of the solvent chambers has a total volume of $10 \mu \mathrm{L}$ and the sample chamber is $4 \mu \mathrm{L}$. This is more than sufficient for our typical separation requirements (e.g., 30-60 min gradients at a constant flow rate of $\sim 100 \mathrm{~nL} / \mathrm{min}$ ).

These three pumps feed into a mixer. Our mixer is diffusion based and consists of a long narrow restriction. It has a cross section of $20 \times 20(\mathrm{WxH}) \mu \mathrm{m}^{2}$ and a total length of $2 \mathrm{~mm}$. This narrow channel functions as a mixer by reducing the diffusion lengths necessary for complete mixing.

After the mixer is our column. The column is $100 \times 20(\mathrm{WxH}) \mu \mathrm{m}^{2}$ in cross section and $1.2 \mathrm{~cm}$ long. There is an opening which we can use to pack beads into the column. This column is designed to be packed using conventional slurry methods. The cross section of our column is comparable to the smaller diameter capillary columns commonly used today. Our column is much shorter than commercial columns used today, but it is sufficient to demonstrate the concept. Currently, column length is mainly limited by how well that packaging responds to the higher pressures needed with longer columns.
Finally, after the column we have a freestanding parylene nozzle which extends $1.5 \mathrm{~mm}$ past the end of the chip which we use to couple with a MS.

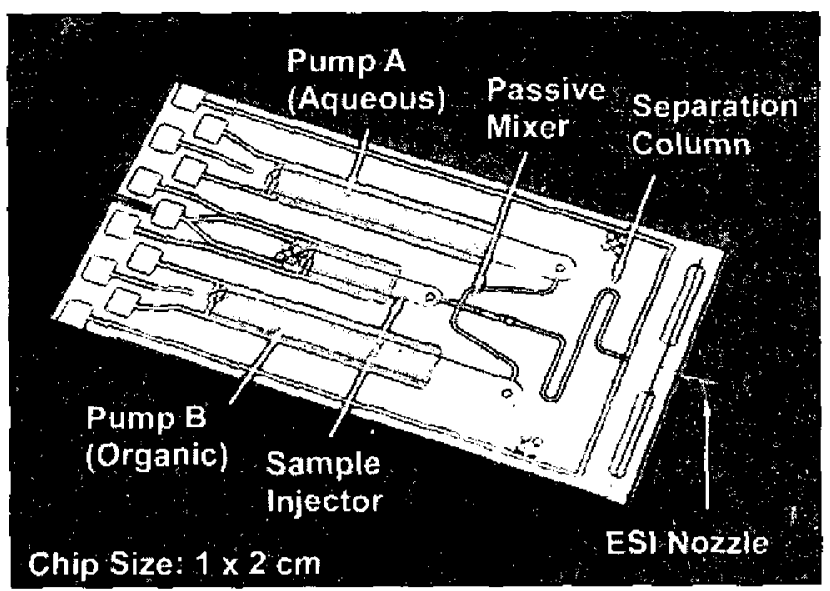

Figure 3: A photograph of the fabricated chip. Note the common features between the chip and the system depicted in Figure 2.

\section{FAbRicAtion}

The chips are fabricated by combining several polymer micromachining technologies. The pump chambers are formed using SU-8 and PDMS while the mixer, the column and nozzle are all realized using parylene. The fabrication process is shown in Figure 4.

First, Ti/Pt is deposited and patterned to form the electrodes. The electrodes used for electrolysis are a pair of interdigitated electrodes with width/spacing of 50/50 $\mu \mathrm{m}$. There are also electrodes at the base of the nozzle which are used to facilitate electrospray from the nozzle. Next, the oxide is patterned and the exposed $\mathrm{Si}$ is roughened using $\mathrm{XeF}_{2}$. This roughened $\mathrm{Si}$ serves as an anchor for the parylene. This is necessary to make sure the column and channels are able to withstand high pressures. The adhesion of parylene to either oxide or bare silicon would be inadequate for our application. Next, a $4 \mu \mathrm{m}$ parylene layer is deposited and pattered using oxygen plasma. This bottom layer forms the bottom of the nozzle. Next, a $20 \mu \mathrm{m}$ sacrificial photoresist layer is spun on. A double exposure reduces the photoresist height in some areas to $5 \mu \mathrm{m}$. This reduction in height is necessary to form the filter structures. After a final $4 \mu \mathrm{m}$ parylene deposition and patterning, a $50 \mu \mathrm{m}$ SU-8 layer is spun on the chip and patterned to strengthen the parylene structures and also provide a planarized surface for further packaging. The sacrificial photoresist is then dissolved and $\mathrm{XeF}_{2}$ used to undercut the nozzle. The chips are broken along the $\mathrm{XeF}_{2}$ etched trench to make the nozzle completely freestanding. 


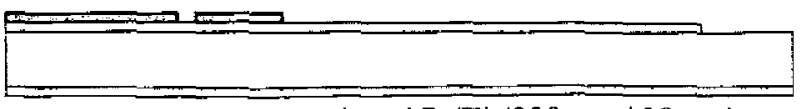

1. Pattern oxide $(1.5 \mu \mathrm{m})$ and $\mathrm{Pt} / \mathrm{Ti}(200 \mathrm{~nm} / 30 \mathrm{~nm})$ electrolysis electrodes.

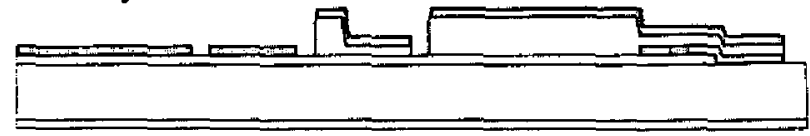

2. Pattern $1^{\text {st }}$ parylene $(4 \mu \mathrm{m})$, sacrificial photoresist ( 20 $\mu \mathrm{m}$ and $5 \mu \mathrm{m})$ and $2^{\text {nd }}$ parylene $(4 \mu \mathrm{m})$ to form the nozzle.

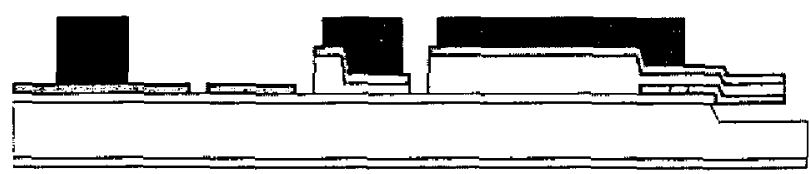

3. Pattern SU-8 $(50 \mu \mathrm{m})$ to planarize the surface, $\mathrm{XeF} 2$ etching to release the nozzle.

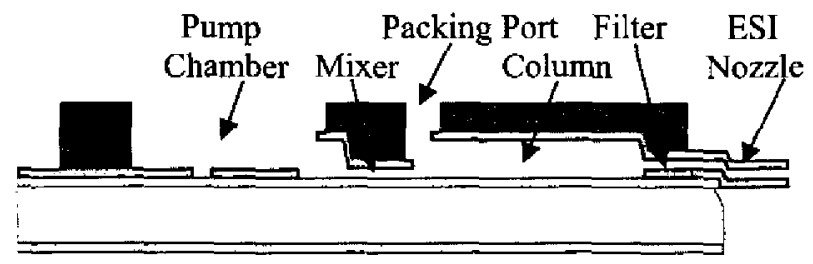

4. Release sacrificial photoresist and free the overhanging nozzle.

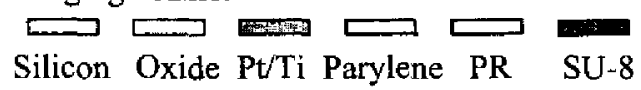

Figure 4: Fabrication process flow.

Further packaging is needed to form the pump chambers. The packaging is illustrated in Figure 5. The first layer in the packaging is a $500 \mu \mathrm{m}$ PDMS gasket. The second layer is a Si piece which has been etched through to further increase the volume of the chambers. Finally, a top PDMS piece is placed over the $\mathrm{Si}$, sealing the chamber. Prior to chip operation, the necessary solvents and sample are injected into their respective chambers using a syringe. A glass cover piece is placed over the packaged chip and the entire structure is then clamped together and positioned in front of the MS.

One very important parameter in designing this chip was finding materials which are compatible with the sample and the organic solvents commonly used in HPLC. All our channels, column, and nozzle are fabricated using parylene. Parylene is an ideal material because of its excellent chemical compatibility. It is also impermeable to most liquids and gases. To strengthen our parylene channels and for packaging purposes, SU-8 is also used. SU-8 has been demonstrated as a possible material for use MS analysis [6] and is widely considered to be compatible with most organic solvents. We also use PDMS as a sealing material. PDMS is generally not used with organic solvent because of the swelling [7]. Seals made from PDMS are adequate for the pressures we expect to encounter though. PDMS is likely to have impurities leaching out of it which could contribute to background noise. To prevent this, all PDMS is soaked for several weeks in ethanol to try to remove these leachables.
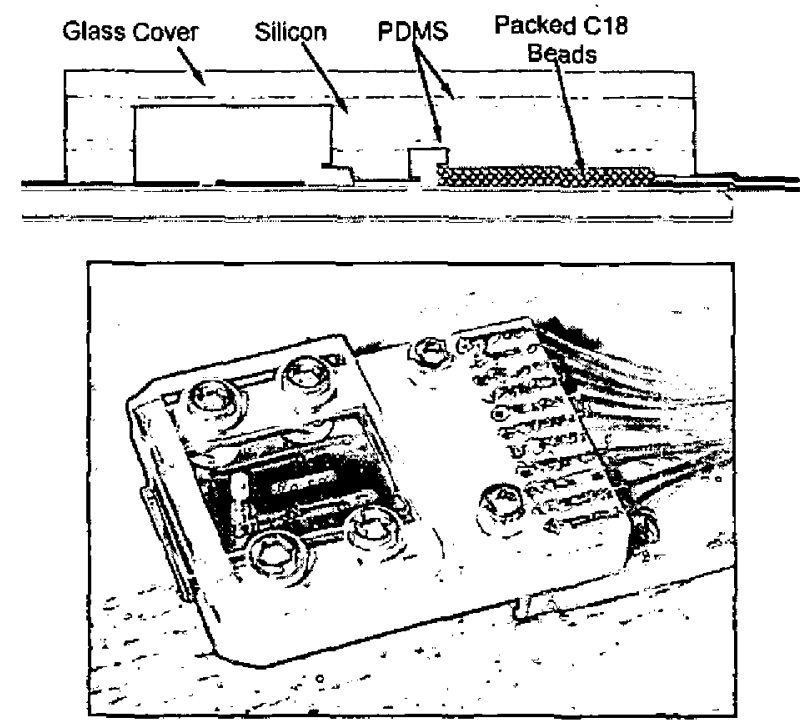

Figure 5: A diagram of the packaging of the chip as well as a picture of the packaged chip. Note the bubbles formed inside chambers.

\section{Results}

The column is slurry-packed with $5 \mu \mathrm{m} \mathrm{C}_{18}$ beads before packaging. $5 \mu \mathrm{m} \mathrm{C}_{18}$ beads are mixed with IPA and mixed vigorously. The beads solution is then forced into the column at high pressure ( 100 psi).

Separation of a Bovine Serum Albumin (BSA) digest is presented. Figure 6 shows the MS output as well as the control current profiles used for the pumps. In this separation, the aqueous chamber was filled with a 95/5/0.1 (water/acetonitrile/formic acid $\mathrm{v} / \mathrm{v}$ ) solution while the organic chamber was filled with a $50 / 50 / 0.1$ solution. The sample chamber contained a $1 \mathrm{pmol} / \mu \mathrm{L}$ sample of BSA digest. The total flow rate during the gradient is estimated to be $100 \mathrm{~nL} / \mathrm{min}$ with pressures inside the chambers reaching $60 \mathrm{psi}$. $500 \mathrm{fmol}$ of sample was injected. The current profile was determined experimentally.

Figure 7 shows the same separation done using a commercial HPLC system and column. $500 \mathrm{fmol}$ of sample was used and the gradient goes from $2 \%$ to $60 \%$ organic at a rate of $1 \% / \mathrm{min}$. The column is packed with 3 $\mu \mathrm{m} \mathrm{C}_{18}$ beads and has a diameter of $75 \mu \mathrm{m}$ and is $10 \mathrm{~cm}$ long. The difference in separation quality is mainly attributed to the shorter column length on our chip (1.2 vs. $10 \mathrm{~cm}$ ). Our chip performs similarly in terms of coverage of the BSA protein, obtaining $50 \%$ coverage compared to $60 \%$ coverage for the commercial system. Total power consumption is very low given the high efficiency of 
pumping with electrolysis. The combined power consumption of the pumps during chip operation is generally around $1 \mathrm{~mW}$.

We have also demonstrated the ability to do multiple separations of the sample without refilling of the chambers and have achieved separations using other solvent systems. Also, no significant sources of noise from the chip have been found

\section{CONClusion}

We have demonstrated the first fully integrated LC-ESI system on a chip. We integrated (gradient and sample injection) pumps, a mixer, a column, filters, and an ESI nozzle to create a functional device. Current work involves integrating sensors for feedback control and improving separation performance.

\section{REFERENCES}

[1] T. Laurell, G. Marko-Varga, "Miniaturisation is Mandatory Unraveling the Human Proteome," Proteomics, vol. 2, pp.345-351, 2002.

[2] N. Lion, T. C. Rohner, L. Dayon, I. L. Arnaud, E. Damoc, N. Youhnovski, Z. Y. Wu, C. Roussel, J. Josserand, H. Jensen, J. S. Rossier, M. Przybylski, H.H. Girault, "Microfluidic Systems in Proteomics," Electrophoresis, vol. 24, pp. 3533-3562, 2003.

[3] K. Killeen, H. Yin, R. Brennen, T. van de Goor, "Multidimensional Chromatography and Digestion using HPLC-CHIP/MS," Proc. $\mu$ TAS 2004, vol. 2, pp. 542-544.

[4] Q. He, C. Pang, Y.C. Tai, T.D. Lee, "Ion Liquid Chromatography On-a-Chip with Beads-Packed Parylene Column," Proc. MEMS 2004, pp. 212-215.

[5] J. Xie, Y. Miao, J. Shih, Q. He, J. Liu, Y.C. Tai, T.D. Lee, "An Electrochemical Pumping System for OnChip Gradient Generation," Analytical Chemistry, vol. 76, pp. 3756-3763, 2004.

[6] S. Arscott, S. Le Gac, C. Druon, P. Tabourier, C. Rolando. "A Planar On-Chip Micro-Nib Interface for NanoESI-MS Microfluidic Applications. "Journal of Micromechanics and Microengineering, vol 14, pp. 310-316. 2004.
[7] J.N. Lee, C. Park, G.M. Whitesides. "Solvent Compatibility of Poly(dimethysiloxane)-Based Microfluidic Devices." Analytical Chemistry, vol 75, pp. 6544-6554, 2003.

\section{ACKNOWLEDGEMENTS}

This work is supported in part by NSF ERC Center at Caltech (EEC-9402726) and NIH through grant 5R01 RR06217-10.
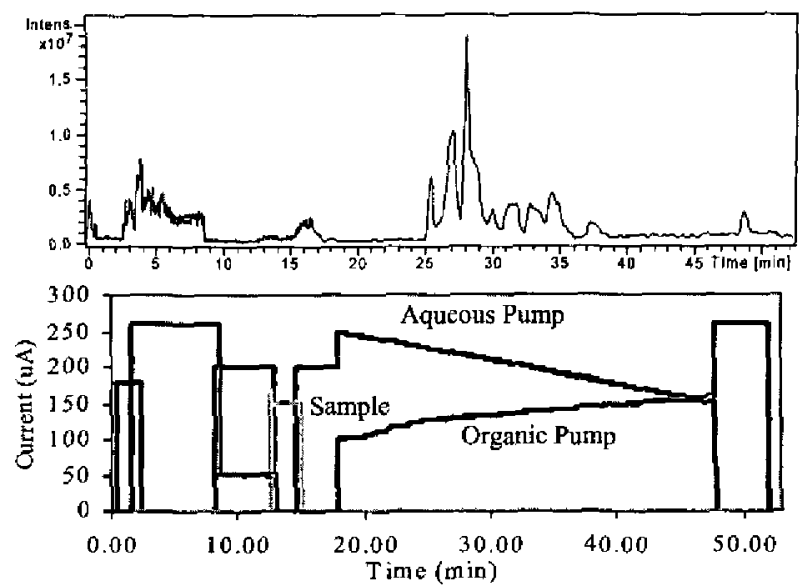

Figure 6: Separation of a BSA digest using our chip as well as the current profiles for the pumps. (Note the distinct regions in the separation. 2-9 min.: Rinse with organic to clean column. 9-15 min.: Column equilibration. $15-17$ min.: Sample injection. $18-48$ min.: Gradient. 48 min.-end: Final column cleaning.)

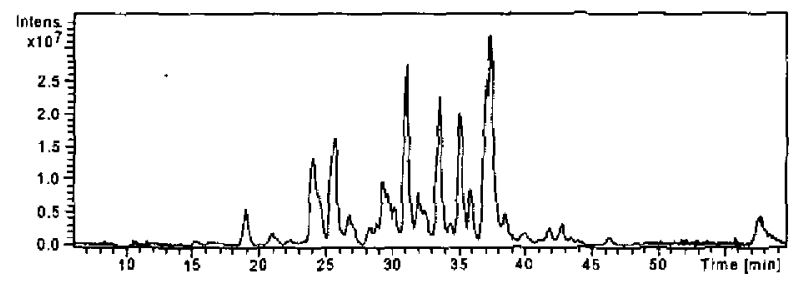

Figure 7: Separation of BSA Digest using commercial HPLC system. 